\title{
Are Most Published Research Findings False In A Continuous Universe?
}

Kleber Neves ${ }^{1}$, Pedro B. Tan ${ }^{2,3}$ and Olavo B. Amaral ${ }^{1}$

\footnotetext{
${ }^{1}$ Institute of Medical Biochemistry Leopoldo de Meis, Federal University of Rio de Janeiro. Rio de Janeiro, Brazil

${ }^{2}$ Faculty of Science, Vrije Universiteit Amsterdam, Amsterdam, The Netherlands

${ }^{3}$ Faculty of Science, Universiteit van Amsterdam, Amsterdam, The Netherlands
}

Kleber Neves https://orcid.org/0000-0001-9519-4909

Pedro B. Tan https://orcid.org/0000-0001-9790-9568

Olavo B. Amaral https://orcid.org/0000-0002-4299-8978

\section{Corresponding author:}

Kleber Neves

E-mail: kleber.neves@bioqmed.ufrj.br

\begin{abstract}
Diagnostic screening models for the interpretation of null hypothesis significance test (NHST) results have been influential in highlighting the effect of selective publication on the reproducibility of the published literature, leading to John Ioannidis' much-cited claim that most published research findings are false. These models, however, are typically based on the assumption that hypotheses are dichotomously true or false, without considering that effect sizes for different hypotheses are not the same. To address this limitation, we develop a simulation model that overcomes this by modeling effect sizes explicitly using different continuous distributions, while retaining other aspects of previous models such as publication bias and the pursuit of statistical significance. Our results show that the combination of selective publication, bias, low statistical power and unlikely hypotheses consistently leads to high proportions of false positives, irrespective of the effect size distribution assumed. Using continuous effect sizes also allows us to evaluate the degree of effect size overestimation and prevalence of estimates with the wrong signal in the literature, showing that the same factors that drive false-positive results also lead to errors in estimating effect size direction and magnitude. Nevertheless, the relative influence of these factors on different metrics varies depending on the distribution assumed for effect sizes. The model is made available as an R ShinyApp interface, allowing one to explore features of the literature in various scenarios.
\end{abstract}




\section{Introduction}

In 2005, John Ioannidis published an article with the alarming conclusion that most published findings in biomedical science are false (Ioannidis, 2005). The work, which has received thousands of citations since then, was a turning point for the awareness of reproducibility issues in science, which has steadily increased over the last two decades in a process that has been viewed both as a "reproducibility crisis” and as a “credibility revolution” (Baker, 2016; Jamieson, 2018).

The paper's conclusion is based on an analytical model of the scientific discovery process, largely inspired by the framework used in the interpretation of clinical diagnostic tests (for historical references, see Mayo \& Morey, 2017). In this framework, hypotheses are either true or false, and scientists testing them find positive or negative results on the basis of null-hypothesis significance tests. The results will thus depend both on the proportion of true to false hypotheses and on the error rates of the tests, which are combined using Bayes' theorem. Assuming values for the model's parameters in different scenarios of biomedical research, Ioannidis calculates that the positive predictive value (PPV; the proportion of true positives among positive results; see Methods for details) of the published literature would be below $50 \%$ in most cases.

Despite its broad influence, the study has also attracted a fair amount of criticism. Critics of the model have pointed out its pessimistic assumptions about the prevalence of bias (Goodman \& Greenland, 2007) and the low proportion of true hypotheses assumed for some scenarios (Bird, 2018; Stewart \& Plotkin, 2020). After all, even with well-powered and unbiased research, one can still get a very low PPV with a low enough prevalence - with the converse also being true, as long as the prevalence is high enough (Mayo \& Morey, 2017). The model also makes implicit - and pessimistic - assumptions about publication bias and interpretation of individual findings in isolation that do not necessarily hold for all fields of science. By exploring the effects of removing some of these assumptions, other authors have found a less dreary picture of the literature (Moonesinghe et al., 2007; Steinfath et al., 2018; Ulrich et al., 2020).

A more basic concern, however, is that the model makes unrealistic assumptions about the nature of scientific hypotheses, which are treated as dichotomously true or false (Mayo \& Morey, 2017). In a real-life scenario, however, it makes no sense to consider statistical power as constant for all true effects, which essentially assumes that they all have the same size, or consider that all false hypotheses have effect sizes strictly equal to zero. On the contrary, effect sizes in most fields are likely to follow a continuous distribution, (Meehl, 1978; Orben \& Lakens, 2020; Wilson et al., 2020), and while NHSTs are designed to detect scientifically relevant effects, it is not the case that all relevant effects are of similar magnitude, or that all other effects are null (Gelman \& Carlin, 2014). As Wilson and colleagues (2020) put it, the framework is at most a useful fiction that can mislead if misused. 
To address these criticisms and investigate whether the conclusions of Ioannidis (2005) hold true in more realistic scenarios, we developed a simulation model that expands upon their original premises. Our model assumes continuous effect sizes following different distributions, which are classified as true or false based on minimum effect sizes of interest. Besides evaluating positive predictive values, we also analyze other measures of accuracy, such as signal and magnitude errors (Gelman \& Carlin, 2014), under different scenarios of prevalence, statistical power and bias. The model is available as an $\mathrm{R}$ ShinyApp interface that allows the user to explore features of the literature under various assumptions about the scientific discovery process.

\section{Results}

The different effect size distributions used in the model are shown in Figure 1. We initially use a dichotomous distribution of hypotheses as in Ioannidis (2005), with explicit effect sizes for true and false hypothesis (0 and 1, respectively; Dichotomous model, Figure 1A). In each experiment, scientists perform t-tests between two experimental groups. An effect size of 0 or 1 is selected randomly for each experiment with a probability based on the prevalence of true hypotheses. Samples are then generated from normal distributions centered at zero (control) or at the effect size (treatment), both with SD $=1$ to reflect sampling error, with a sample size calculated to yield a given statistical power to detect a typical true effect. The difference between group means is used as the effect size estimate for each experiment, and results are published if significant results $(\mathrm{p}<0.05)$ are found. When results are non-significant, results have a probability of being biased according to a bias parameter. If results are biased, the experiment is repeated until a significant result is obtained and published. Simulations are performed until the literature contains 5,000 results.

The positive predictive value (PPV) of published findings is calculated for this dichotomous distribution under different combinations of statistical power, bias and prevalence of true effects (Figure 2A). Increasing statistical power leads to higher PPVs, as more true effects are detected by NHSTs. Increasing bias, on the other hand, markedly lowers the PPV, especially at lower prevalences, as more false positives are generated when bias is prevalent. Our simulation results are in line with those obtained analytically by Ioannidis (2005) - for a more direct comparison, the same curves expressing the likelihood of true hypotheses in odds (as in the original publication) are shown in Figure S1. 
A Dichotomous

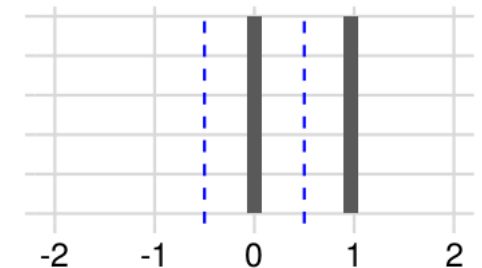

B

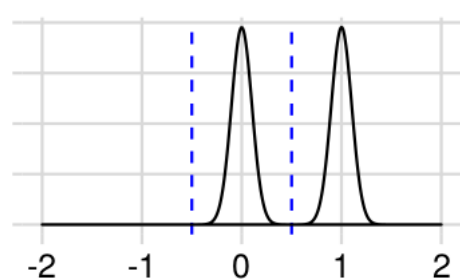

C Overlapping Peaks

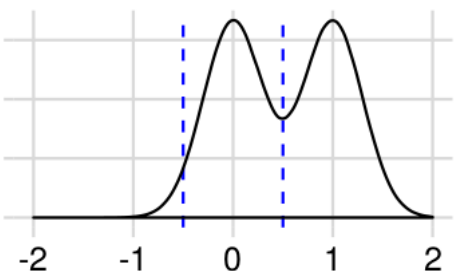

\section{Two Normals}

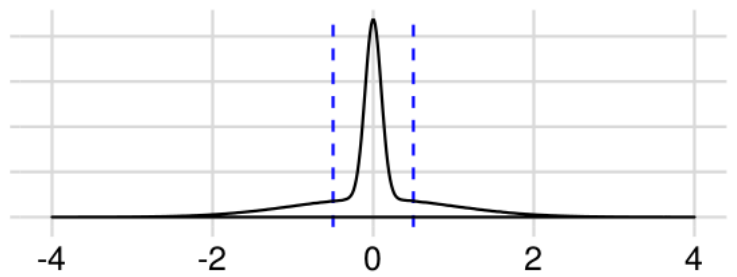

\section{E Single Normal}

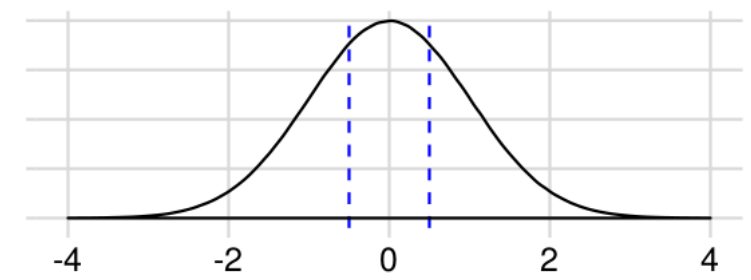

Figure 1: Effect size distributions used in the simulations. In the Dichotomous model (A), the density curve is replaced by a histogram, as effects sizes are strictly 0 or 1 . The Two Peaks (B), Overlapping Peaks (C) and Two Normals (D) models are built as a mixture of two normal distributions with different means and/or SDs (see Methods for details). The weight parameter that regulates the mixture is 0.5 in the examples shown, leading to equal weights for both curves - in simulations, however, it varies according to the prevalence of true effects. For the Single Normal model (E), the weight parameter equals 1 and the standard deviation is used to control the prevalence. Dashed lines indicate the minimum effect size of interest (held fixed at 0.5 for all simulations): effects are considered true if their absolute size is larger than this threshold.

A dichotomous distribution containing only null and large effects makes it trivial to define true and false hypotheses; in reality, however, a real scientific field is likely to have an unknown distribution of continuous effect sizes. To model this, we used different mixtures of normal distributions, varying their spreads, centers and relative contribution to the population of effects. To distinguish true from false effects in this scenario, one must introduce a minimum effect size of interest, above which hypotheses are considered true. This parameter can be interpreted as the effect size that would be considered relevant to support a given hypothesis, and is set at 0.5 for all simulations. Sample size calculations in this case are performed to detect an average true effect, defined as the mean of all true effects (i.e. those above the minimum of interest) within the distribution. That said, in accordance with typical practices in most scientific fields (at least within the biomedical sciences), statistically significant results are published as positive even if their estimate is below the minimum effect size of interest (although this only happens when statistical power is high).

We first expand our initial model to a Two Peaks model (Figure 1B), which maintains the dichotomy between null and true effects, but includes some variation in effect sizes. In this scenario, effect sizes are drawn from Gaussians centered at 0 or 1 with an SD of 0.1 . The relative 
weight of each curve is used to vary the prevalence of true effects. PPV curves for two peaks are similar to the dichotomous model (Figure 2B), as most effects are still close to 0 or 1 , with negligible chances of crossing the minimum effect of interest threshold. We then proceed to an Overlapping Peaks model (Figure 1C), which uses a larger SD of 0.3 for Gaussians centered at 0 and 1 and leads to a larger overlap between distributions. In this case, PPVs are slightly lower than in the two peaks model, particularly when power is high, as small effects under the minimum effect size of interest are eventually detected as positive; nevertheless, the overall results are still quite similar to those of the previous models (Figure 2C).

Even though it is a more plausible scenario than the Dichotomous model, modeling effect sizes with peaks still seems unrealistic, as both the Two Peaks and Overlapping Peaks models are arbitrarily enriched in large effects around 1. A more plausible effect size distribution is a normal centered at zero, forming a continuous distribution where most effects sizes are small, and larger effects are found with a prevalence that varies with the standard deviation. Simulations in this case are initially performed with two normals centered at zero, with SDs of 0.1 and 1 (Two Normals model, Figure 1D). In this case, the prevalence of true effects is still adjusted by changing the relative weight of both normals while maintaining the SDs constant. We then move on to simulations using a Single Normal model (Figure 1E), in which the prevalence of true effects is controlled by varying the standard deviation. PPVs are smaller in these cases, as centering distributions around zero causes effects slightly under the minimum effect size of interest to be more frequent than those above it (Figure 2D and 2E). This in turn leads false-positive effects to be more common than when two peaks are used. Regardless of the effect size distribution, increasing power leads to a greater PPV, while higher bias decreases it.

In all of these scenarios, the decision to publish does not take into account the minimum of interest - i.e. a significant result with a magnitude lower than 0.5 is still published. We do believe this is a realistic assumption in the biomedical sciences, in which sample size calculations or discussions about effect sizes tend to be scarce (e.g. Carneiro et al., 2018). That said, as shown in Figure S2, including effect sizes in publishing decisions changes the results only when power is high, as estimates below the minimum effect of interest will not be significant if the power to detect this effect (which is smaller than those used for sample size calculations) is under $50 \%$. 


\section{A Dichotomous}

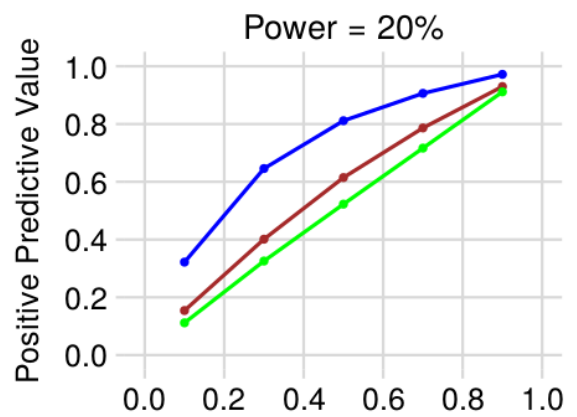

Power $=50 \%$

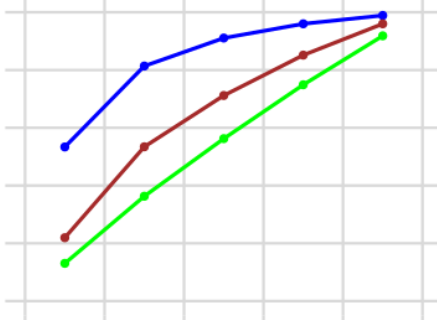

$\begin{array}{llllll}0.0 & 0.2 & 0.4 & 0.6 & 0.8 & 1.0\end{array}$

Prevalence of True Effects
Power $=80 \%$

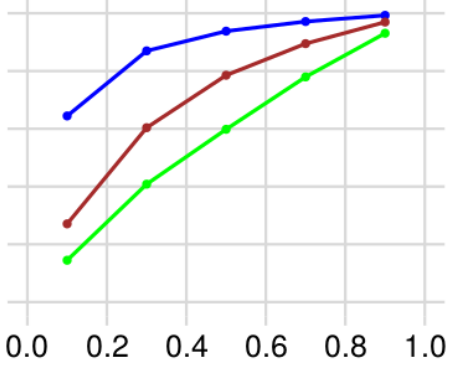

Bias

$\rightarrow 0$

$\rightarrow 0.2$

$\rightarrow 0.6$

\section{B Two Peaks}
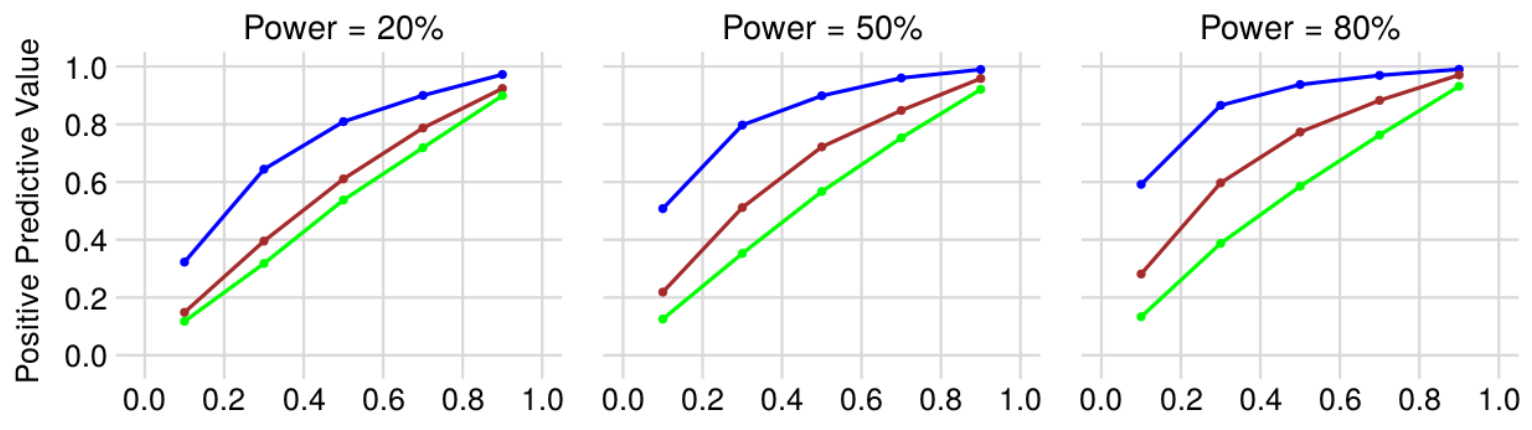

Bias

$\rightarrow 0$

$\rightarrow 0.2$

$\rightarrow 0.6$

\section{Overlapping Peaks}

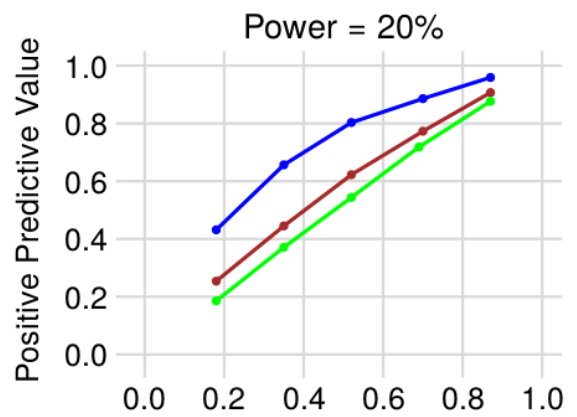

Power $=50 \%$

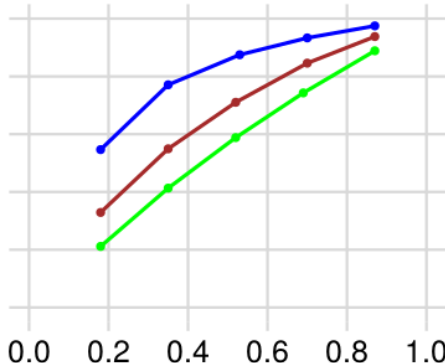

Prevalence of True Effects
Power $=80 \%$

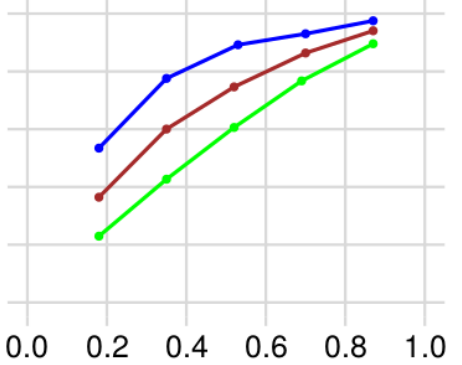

Bias

$\rightarrow 0$

$\rightarrow 0.2$

$\rightarrow 0.6$

\section{Two Normals}

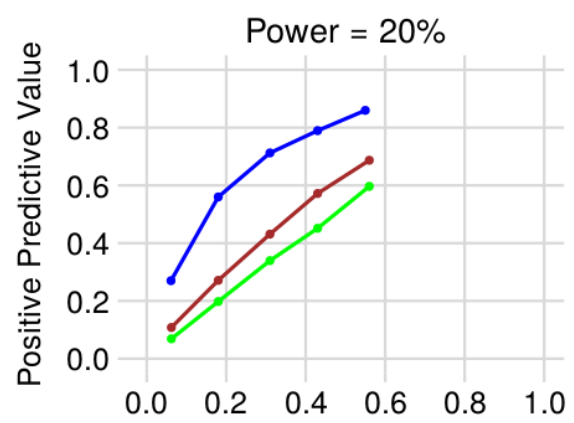

Power $=50 \%$

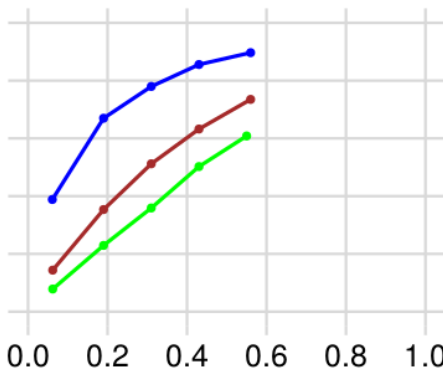

Prevalence of True Effects

\section{E Single Normal}
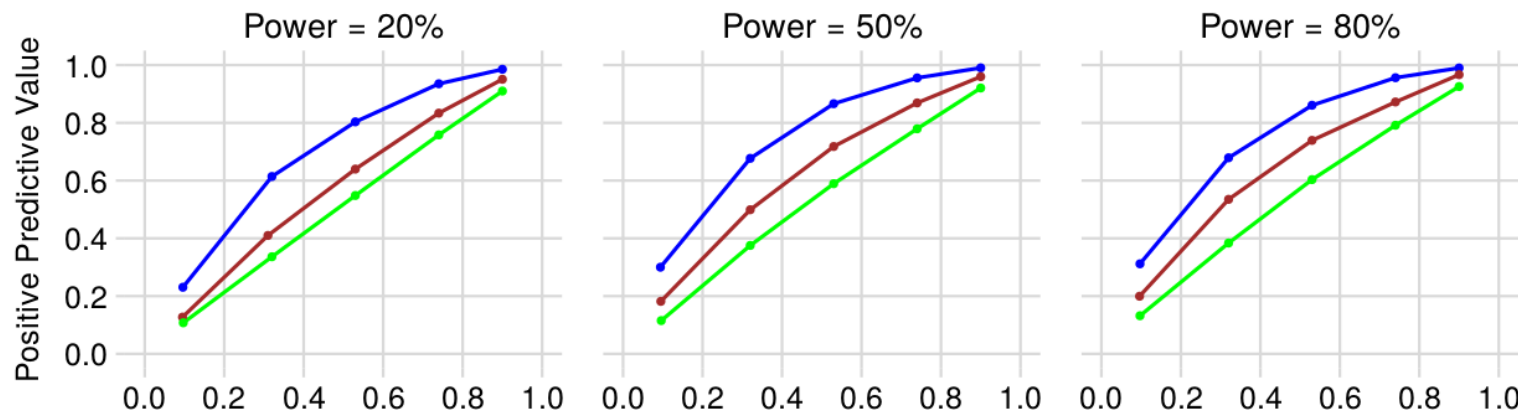

Bias

$\rightarrow 0$

$\rightarrow 0.2$

$\rightarrow 0.6$ 
Figure 2. PPV as a function of the prevalence of true effects, statistical power and bias for (A) Dichotomous, (B) Two Peaks, (C) Overlapping Peaks, (D) Two Normals and (E) Single Normal effect size distributions. Each point corresponds to a simulation with 5,000 published findings. True effects are defined as those above the minimum of interest (Cohen's $d>0.5$ in all simulations). In each panel, graphs correspond to 20\%, 50\% and 80\% power from left to right. Blue, red and green curves correspond to bias (i.e. prevalence of negative results that are repeated until becoming positive and then published) of 0, 0.2 and 0.6, respectively. Prevalence ranges vary among scenarios, as for some distributions some prevalences cannot be achieved without changing other parameters: in the Two Normals model in particular, one cannot obtain a prevalence above $62 \%$ unless either the SD of the wider normal or the minimum effect of interest is changed.

PPVs for the different models using the same parameter scenarios used in Ioannidis (2005) are shown in Table 1. Results for the dichotomous model are very close to the analytical predictions, confirming the validity of the simulations (for the actual prevalences obtained in each simulation, see Table S1). For the Single Normal model, the PPV is lower than in the Dichotomous or Two Peaks distributions, as a normal centered at 0 has a higher mass of effects just below the minimum of interest. These effects will often be estimated above the critical value for significance and become false positives, especially when power is high, which makes them more likely to be detected.

\begin{tabular}{|c|c|c|c|c|c|c|c|c|c|c|}
\hline Scenario description & Power & $\begin{array}{l}\text { Odds for } \\
\text { True } \\
\text { Effects }\end{array}$ & $\begin{array}{l}\text { Prevalence } \\
\text { of True } \\
\text { Effects }\end{array}$ & Bias & Analytical & Dichotomous & Two Peaks & $\begin{array}{l}\text { Overlapping } \\
\text { Peaks }\end{array}$ & Two Normals & Single Normal \\
\hline Adequately powered RCT with little bias and $1: 1$ pre-study odds & $80 \%$ & $1: 1$ & $50 \%$ & $10 \%$ & $85 \%$ & $85,0 \%$ & $84,4 \%$ & $79,7 \%$ & $79,3 \%$ & $76,0 \%$ \\
\hline Confirmatory meta-analysis of good quality RCTs & $95 \%$ & 2:1 & $66,7 \%$ & $30 \%$ & $85 \%$ & $85,4 \%$ & $85,4 \%$ & $81,1 \%$ & $\stackrel{*}{ }$ & $80,5 \%$ \\
\hline Meta-analysis of small inconclusive studies & $80 \%$ & 1:3 & $25 \%$ & $40 \%$ & $41 \%$ & $39,9 \%$ & $40,4 \%$ & $37,7 \%$ & $38,2 \%$ & $36,4 \%$ \\
\hline Underpowered, but well-performed phase I/II RCT & $20 \%$ & 1:5 & $16,7 \%$ & $20 \%$ & $23 \%$ & $24,0 \%$ & $24,8 \%$ & $23,9 \%$ & $25,4 \%$ & $22,3 \%$ \\
\hline Underpowered, poorly performed phase I/II RCT & $20 \%$ & 1:5 & $16,7 \%$ & $80 \%$ & $17 \%$ & $17,3 \%$ & $17,2 \%$ & $17,4 \%$ & $17,9 \%$ & $16,1 \%$ \\
\hline Adequately powered exploratory epidemiological study & $80 \%$ & $1: 10$ & $9,1 \%$ & $30 \%$ & $20 \%$ & $19,9 \%$ & $19,6 \%$ & $17,3 \%$ & $19,0 \%$ & $16,8 \%$ \\
\hline Underpowered exploratory epidemiological study & $20 \%$ & 1:10 & $9,1 \%$ & $30 \%$ & $12 \%$ & $12,5 \%$ & $11,7 \%$ & $12,0 \%$ & $13,6 \%$ & $12,1 \%$ \\
\hline Discovery-oriented exploratory research with massive testing & $20 \%$ & $1: 1000$ & $0,1 \%$ & $80 \%$ & $0,10 \%$ & $0,1 \%$ & $0,1 \%$ & $-* *$ & $0,3 \%$ & $0,1 \%$ \\
\hline $\begin{array}{l}\text { Discovery-oriented exploratory research with massive testing, but with } \\
\text { more limited bias (more stand ardized) }\end{array}$ & $20 \%$ & $1: 1000$ & $0,1 \%$ & $20 \%$ & $0,15 \%$ & $0,2 \%$ & $0,2 \%$ & $-* *$ & $0,2 \%$ & $0,1 \%$ \\
\hline
\end{tabular}

Table 1: Positive predictive value (PPV) for simulated scenarios varying in power, bias and prevalence of true effects (i.e. above the minimum of interest). Scenario descriptions, parameter combinations and the Analytical column are adapted directly from Ioannidis (2005). The remaining columns contain the PPVs for simulations of 5,000 published findings using each of the different effect size distributions in our model. Parameters for the distributions are set so that the prevalence of effects above the minimum of interest approximates the specified odds (see Table S1 for model parameters and achieved prevalences for each scenario). Missing values occur in some scenarios when the desired prevalence cannot be achieved without changing the minimum effect of interest, thus complicating direct comparisons with other scenarios. * For Two Normals, one cannot obtain a prevalence of $66.7 \%$ without changing the minimum effect of interest or the SD of the wider normal, as a normal $\mathrm{N}(0,1)$ has only about $62 \%$ of its mass beyond the minimum of interest on both tails. ** For Two Peaks, one cannot obtain a prevalence of $0.1 \%$ without changing the minimum effect of interest, as a normal distribution $\mathrm{N}(0,0.2)$ has over $1 \%$ of its mass beyond the minimum of interest on both tails. 
Importantly, the PPV for most of these scenarios is below 50\% and falls markedly with decreasing prevalence of true effects and increasing bias. Even without bias, at a very low prevalence a high PPV can only be obtained with a combination of high power (low type II error rate) and a very stringent alpha (low type I error rate). Therefore, irrespective of the distribution, it is difficult to obtain a PPV above $50 \%$ in low prevalence scenarios when Ioannidis' assumptions including full publication bias, isolated publication of findings and use of a fixed significance threshold of $\alpha=0.05$ - hold true. If such practices are indeed prevalent in a given field of science, most published research findings are likely to be false even when continuous effect sizes are assumed.

There are caveats, of course, both in the assumptions and in the dichotomization between true and false hypotheses, which is necessary to calculate positive predictive values in a diagnostic screening framework. In our case, this was achieved by the use of an arbitrary minimum effect of interest threshold to define true studies. In reality, however, accurate estimation of effect sizes can be seen as more important than classifying effects as true or false on the basis of statistical significance (Fidler \& Cumming, 2014; Wilson et al., 2020). We thus also study how our modeled scenarios perform in terms of accuracy in estimating the magnitude of effect sizes.

Moving away from the idea that effects either exist or are practically zero, we analyze two other types of error in our simulations: signal (type $S$ ) errors and magnitude (type $\mathrm{M}$ ) errors (Gelman \& Carlin, 2014). Signal errors occur when the estimated effect size has the opposite sign of the real effect size. Magnitude errors are measured by an exaggeration factor defined as the ratio between the estimated effect size and the true underlying effect, as overestimation is expected when publication decisions rely on statistical significance (Ioannidis, 2008). These errors are only measured in the simulations for published results - i.e. when a significant result is obtained. Of note, Type $\mathrm{S}$ and Type $\mathrm{M}$ errors cannot be defined if the real effect is exactly zero, and therefore are not evaluated in the Dichotomous model.

The type S error rate for the other distributions is presented in Figure 3. Signal errors occur more frequently at lower power, as smaller sample sizes make it more likely that estimates will incorrectly yield significant results in the opposite direction. Bias also increases the signal error rate, as small effects, which are more likely to be estimated with the wrong signal, also become more likely to be published. Increasing the prevalence of true effects decreases the signal error rate, as this leads to a greater representation of large effects whose estimates are unlikely to cross zero. Thus, the error rate approaches 0 as the prevalence approaches 1 , even in the presence of bias. Interestingly, the frequency of type $S$ errors is higher for distributions that are enriched in effects close to 0 , as in the case of the Two Peaks and Two Normals model. For the Overlapping Peaks and 


\section{A Two Peaks}
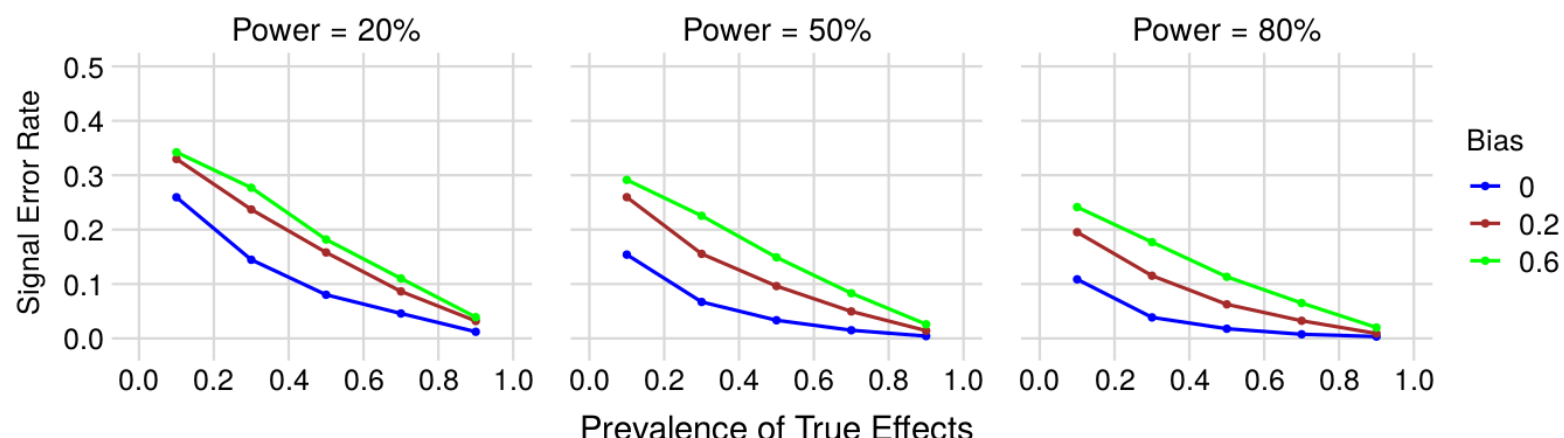

\section{B Overlapping Peaks}
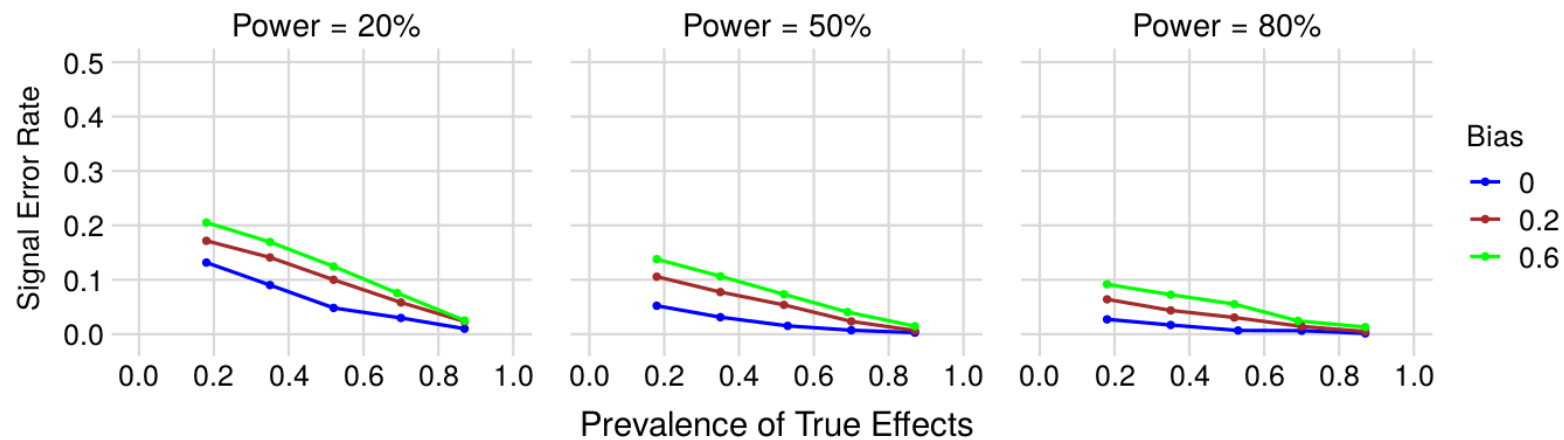

\section{Two Normals}
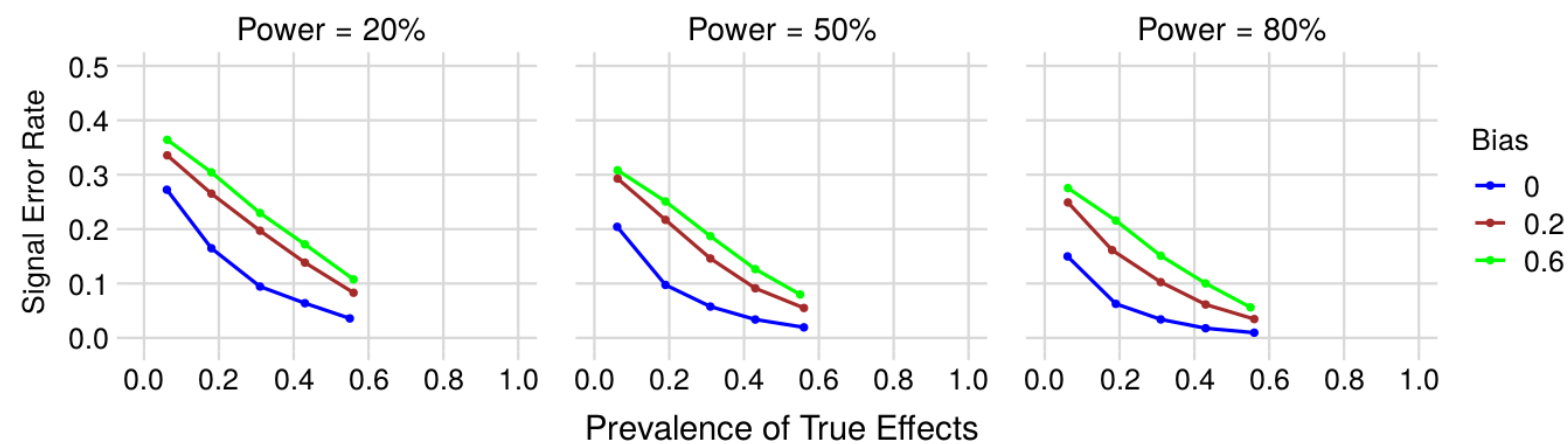

\section{Single Normal}
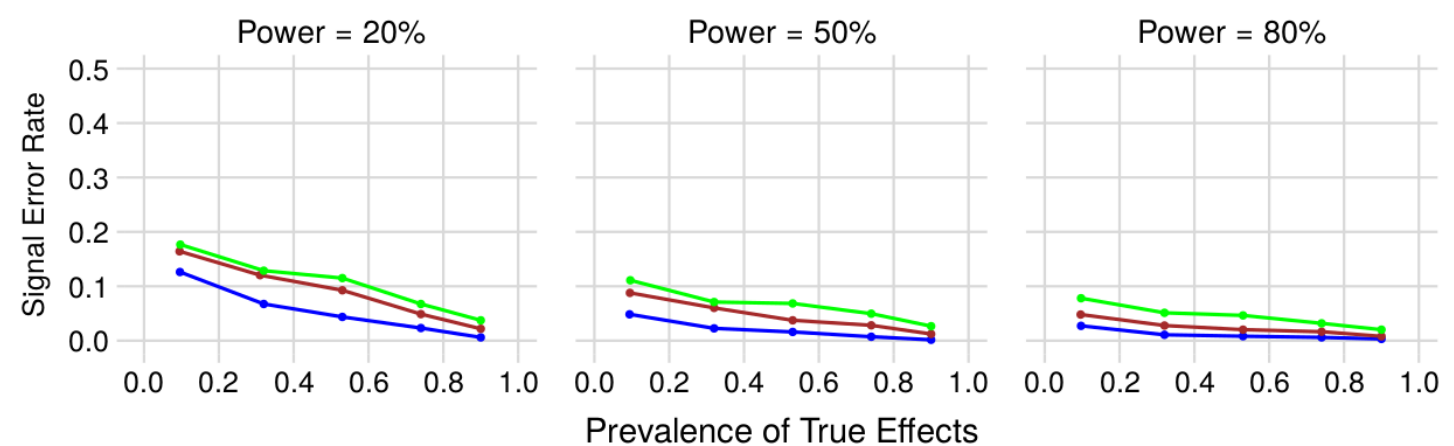

Bias

$\rightarrow 0.2$

$\rightarrow 0.6$

Figure 3: Type $S$ error rate (i.e. published estimates with the wrong signal) as a function of the prevalence of true effects, statistical power and bias for (A) Two Peaks, (B) Overlapping Peaks, (C) Two Normals and (D) Single Normal effect size distributions. The rate corresponds to the percent of published (i.e. statistically significant) estimates that are in the opposite direction of the true effect. Each point corresponds to the signal error rate from a simulation with 5,000 published findings. In each panel, graphs correspond to $20 \%, 50 \%$ and $80 \%$ power from left to right. Blue, red and green curves correspond to bias of $0,0.2$ and 0.6 , respectively. Prevalence ranges vary among scenarios, as for some distributions some prevalences cannot be achieved without changing other parameters. 
Single Normal distributions, a higher fraction of false effects lies close to the minimum of interest therefore, even though these might be incorrectly detected as positive results by effect size inflation, they are unlikely to be estimated with the wrong signal.

Similarly, the exaggeration of effect size estimates (i.e. type $M$ error) is lower with increasing prevalence, increasing power and decreasing bias in all distributions. Increasing power produces better estimates, as larger sample sizes lead to lower variability between samples and smaller type M errors. On the contrary, when power is low, even true effects sometimes require exaggeration caused by sampling error to yield significant results, systematically inflating effect sizes in the literature (Ioannidis, 2008). Type $\mathrm{M}$ error also varies greatly depending on the prevalence of true hypotheses. As sampling error is fixed at 1, large errors are unlikely for large effects, while small effects near zero can result in very large exaggeration ratios. These findings mirror analytical results showing that low signal-to-noise ratios lead to high exaggeration (van Zwet \& Cator, 2020).

Similarly to what is observed for type S errors, as the prevalence of large effects decreases, the higher proportion of small effects drives type $M$ errors up: for the worst-case scenarios in which the false-positive rate is high and distributions are enriched in effects close to 0 (as in the Two Peaks and Two Normals models), the median exaggeration rate can reach values of up to 15 . With high prevalence, on the other hand, this value is close to 1 for all models. These results illustrate that studying small effects with low power while publishing only significant findings can lead to a dramatic distortion of effect size estimations, as discussed by other authors (Gelman \& Carlin, 2014; Ioannidis, 2008). For type S error rate and median exaggeration factors for the specific scenarios proposed by Ioannidis (2005), see Tables S2 and S3.

Importantly, all results are dependent on the effect sizes used for power calculations. Our approach to base our calculations on a typical effect size (i.e. the mean of true effect sizes in a given distribution) means that, in the case of the Single Normal model, in which different prevalences are achieved by varying the SD, the effect size used for the calculations varies with prevalence, as larger prevalences imply larger mean effect sizes. To correct for this, we performed additional simulations using fixed values (either the minimum effect of interest or twice this value) for power calculations in this distribution. The results, shown on Figures S3 to S5, show that our results generally hold in this situation as well, and that using the higher value as a reference leads to even lower PPVs and greater type S and M error rates when prevalence is low. 

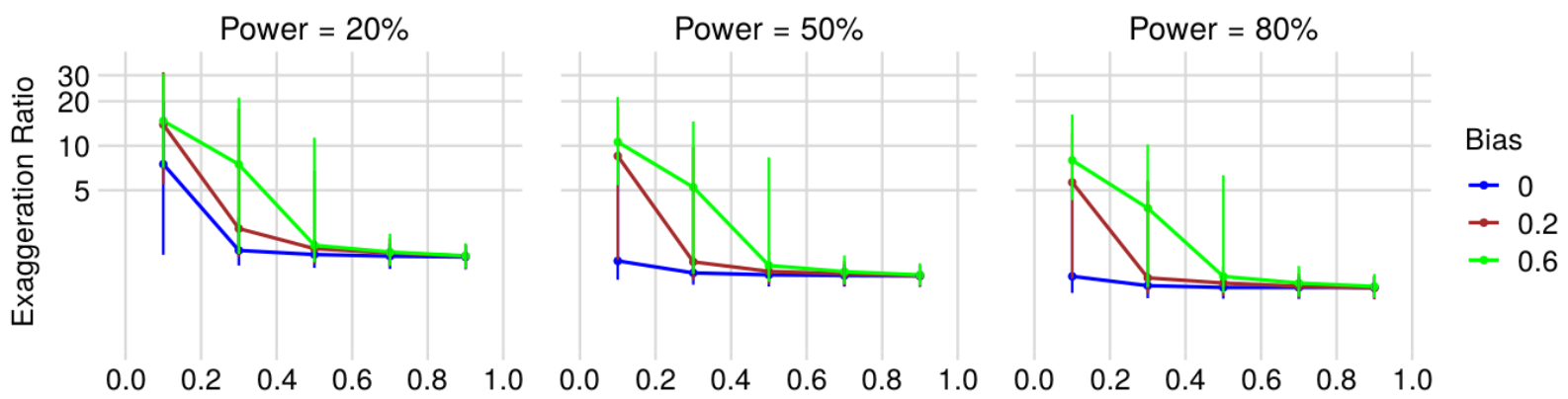

\section{B Overlapping Peaks}
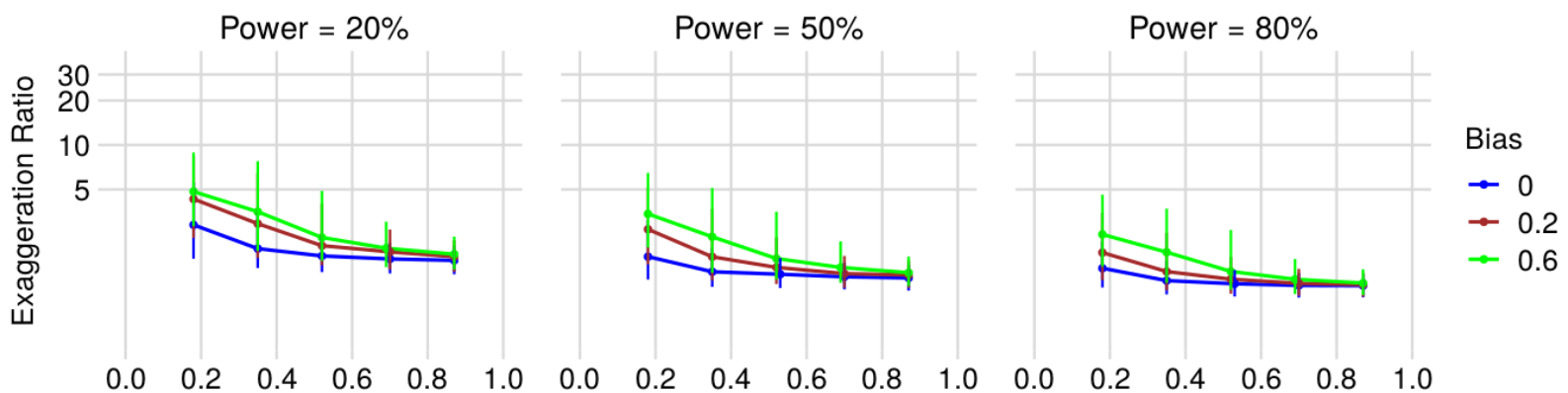

\section{Two Normals}
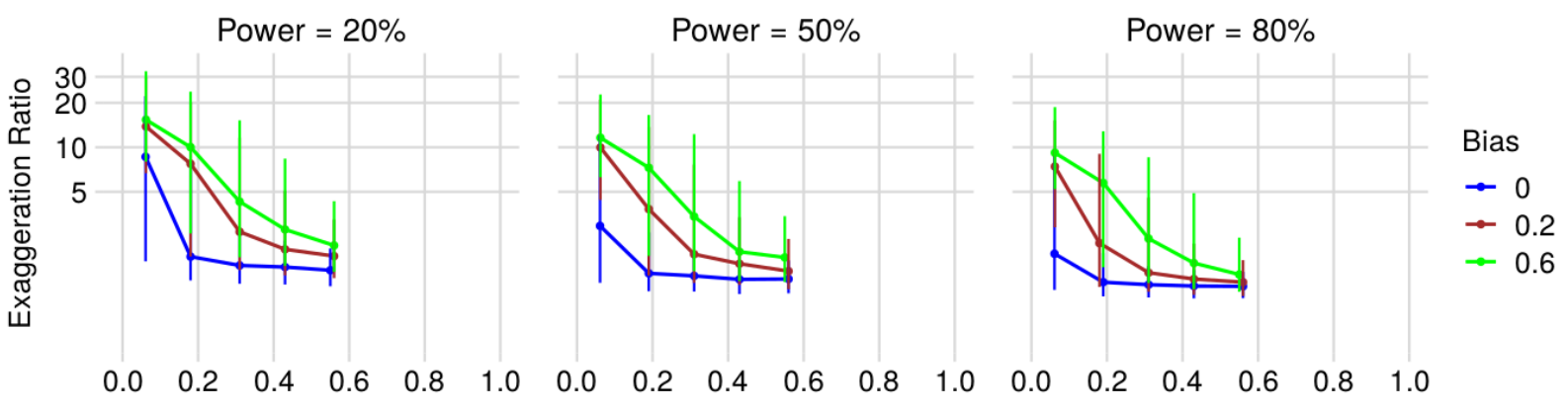

\section{Single Normal}
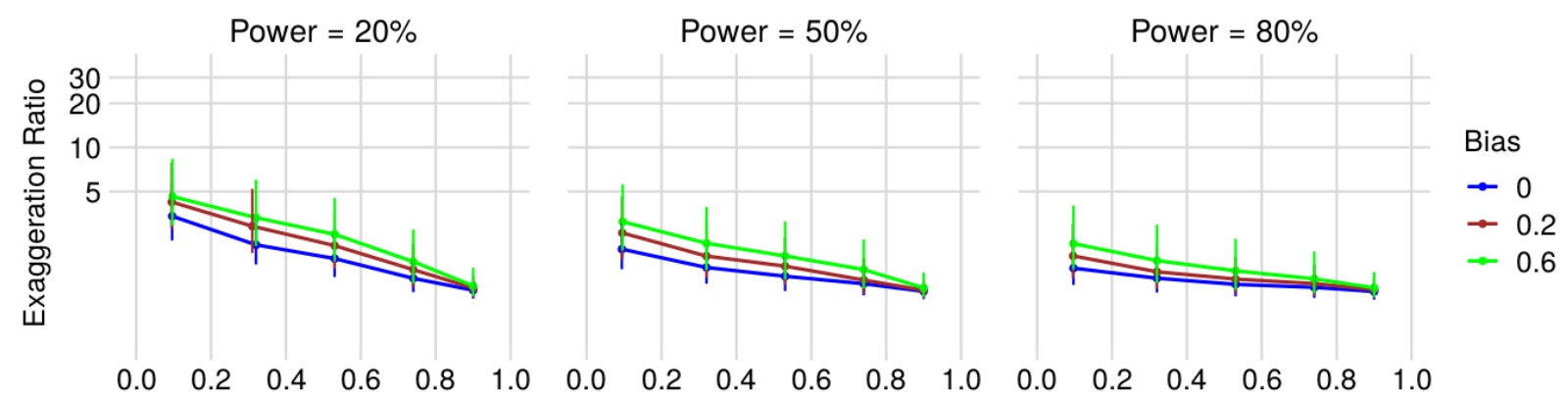

Prevalence of True Effects

Figure 4: Type $M$ error (i.e. effect size exaggeration) as a function of the prevalence of true effects, statistical power and bias for (A) Two Peaks, (B) Overlapping Peaks, (C) Two Normals and (D) Single Normal effect size distributions. Each point corresponds to the median exaggeration ratio for 5,000 published effects in the simulated literature, with error bars indicating the interquartile range. The $\mathrm{Y}$ axis represents the exaggeration ratio in a log scale to allow better visualization. In each panel, graphs correspond to $20 \%, 50 \%$ and $80 \%$ power from left to right. Blue, red and green curves correspond to bias of $0,0.2$ and 0.6 , respectively. Prevalence ranges vary among scenarios, as for some distributions some prevalences cannot be achieved without changing other parameters. 


\section{Discussion}

Our results show that the overall conclusions of the dichotomized model presented by Ioannidis (2005) generally hold for different distributions of continuous effect sizes as well. Depending on features of the literature, most published effect sizes might indeed be false positives i.e. smaller than scientists would judge to be relevant for the field - when low statistical power, high bias and a low prevalence of large effects are combined.

That said, using continuous effect sizes does result in a few differences from the original model. Our results for the specific scenarios in Ioannidis (2005) show that, when power is high, the PPV is lower for continuous distributions than it is in the dichotomous model. This is due to the presence of small effects below the minimum of interest, that can nevertheless result in significant differences when power is high and count as false-positives. This exemplifies a trade-off between sensibility and specificity, and shows that increasing power can have detrimental effects if statistical significance alone is used to judge results (Wilson et al., 2020).

Interestingly, when the focus is switched from classifying effects as true or false to accurately estimating the direction and magnitude of effects, these conclusions are reversed: signal error rates and effect size inflation (i.e. type $\mathrm{S}$ and $\mathrm{M}$ errors) are less problematic in continuous distributions than in dichotomous ones in which a larger fraction of effects are close to 0 . This illustrates a limitation of the diagnostic screening framework, as there is information in an experiment that goes beyond classifying a hypothesis as true or false and will not be captured by the PPV. That said, the combination of low prevalence of true hypotheses, low statistical power and high bias has a detrimental effect both on the PPV and on type S and M errors, once again suggesting that the main conclusions drawn by Ioannidis (2005) hold true in an estimation framework as well.

One way of seeing the results of such models is to consider that science has two steps: one of signal detection, where using significance testing as modeled here is more appropriate, and a second phase of estimation and understanding, when statistical significance alone cannot provide accurate guidance (Gelman, 2018; Wilson et al., 2020). Within this logic, the problem is when both approaches are merged into a single step and the results of "screening science" are taken as more than provisional. Our model shows that this practice not only leads to false-positive results, but also to serious errors in estimation, as the rates of signal and magnitude errors are also affected by statistical power and bias. Even in the absence of bias, median effect sizes can be markedly exaggerated if studies are underpowered, reinforcing commonly voiced concerns about sample size calculations using estimates from the literature (Gelman \& Carlin, 2014; Wilson et al., 2020). It also 
highlights the dangers of the uncritical use of NHSTs, which have been repeatedly pointed out by various authors (McShane et al., 2019; Mayo \& Morey, 2017; Wilson et al., 2020).

Although our simulations address what is perhaps the main methodological shortcoming of the diagnostic screening framework used by Ioannidis (2005), other limitations of the original model still apply to our work. Particularly, its assumptions of absolute publication bias, lack of replication and publication of significant findings irrespective of context are quite pessimistic: the model assumes that scientists will follow the rules of NHSTs blindly, exclusively and invariably publishing significant results with no consideration of effect sizes, prior probabilities or other findings. Although this is certainly an exaggeration, we do believe that excessive reliance on significance tests happens frequently enough for this approximation to be reasonable (KennedyShaffer, 2019). Nevertheless, other results have shown that publication of negative results and replication of published findings can do a lot to mitigate the effect of bias and low power (Moonesinghe et al., 2007; Protzko et al., 2020; Steinfath et al., 2018).

Another limitation added by our framework is the fact that it requires one to set arbitrary minimum effect sizes of interest to correspond to the notion of true and false hypotheses in the diagnostic framework terminology. Although we concede that such dichotomies are artificial, they are needed in order to maintain correspondence with previous models - and with dichotomous views that are still widespread in many areas of science (McShane \& Gal, 2017). Nevertheless, the dissociation between type $\mathrm{S}$ and type $\mathrm{M}$ error rates and false-positive rates for different distributions illustrates the limitations of using any of these approaches in isolation. An additional limitation is that, for continuous models, varying prevalence inevitably requires adjustments in other parameters in the effect size distributions. Thus, prevalence cannot be adjusted independently, and results can be influenced by changes in other parameters.

Concerning more philosophical issues, the difficulties of interpreting the a priori prevalence of true hypotheses in the Ioannidis model (Bird, 2018; Mayo \& Morey, 2017; Wilson et al., 2020) are replaced by concerns about the shape of the underlying distribution of effect sizes in our model. We are partial to arguments made for distributions of the exponential family for effect sizes, centered near zero, with smaller effects being more common than large ones (e.g. Wilson et al., 2020). Nevertheless, we chose to experiment with different distributions to test the sensitivity of the model - from an unrealistically dichotomous one to a continuous distribution with most effects around 0 . In spite of the chosen distribution, the general conclusions concerning false-positive rates in different scenarios seem to hold, albeit with small variations. Nevertheless, even among continuous distributions with exponential tails (e.g. Two Normals and Single Normal), parameters such as the median effect size inflation in the literature were quite sensitive to the exact shape of the 
curve, suggesting that estimates from simulations should be taken with caution in the absence of empirical evidence on effect size distributions.

As future steps, we plan to extend the model to include replications and interlaboratory variation, in order to explore how different criteria for replication track the features of the actual effects, and whether the results of systematic replications can be used to provide empirical estimates of statistical power, bias and prevalence of true hypotheses within a scientific field. This will serve the purpose of informing the development of the analysis plan for the Brazilian Reproducibility Initiative, a multicenter replication of Brazilian biomedical science which we currently coordinate (Amaral et al., 2019), and hopefully provide a reference for interpreting results of large-scale replications in general (Stanley \& Spence, 2014).

\section{Methods}

\section{Model overview}

In our model, scientists pick effects to be tested at random from an underlying distribution of true effect sizes, each of which represents the difference between the population means of a quantitative variable in two groups. Although expressing effect sizes as differences is a common situation in basic biomedical research, they could also be measured in other forms (e.g. correlation coefficients) for similar purposes. Scientists then obtain a sample from each experimental group and compare their means. If the difference is statistically significant, the results are published. If they are not, the result will not be published unless bias is present. The bias parameter dictates the proportion of negative results that artificially reach significance through biased analysis or other means, as in Ioannidis (2005). After a predefined number of findings are published, we can evaluate the accuracy of the literature produced under a given condition by different metrics. We describe the steps and parameters in more detail below.

\section{Effects}

For each iteration of the simulation, an effect is chosen at random from an underlying distribution that represents the effect sizes investigated by scientists within a research field. The effect size distribution in a given field cannot be known in advance, and unbiased data are scarce to provide good estimates, although literature overviews (e.g. Szucs \& Ioannidis, 2017; Carneiro et al., 2018) and systematic replication initiatives (e.g. Nosek et al; 2015) can provide some insights on the subject. Nevertheless, arguments have been made that exponential curves or other distributions 
with most of their density around 0 might be a good approximation, as most effects are likely to be small, with larger effect sizes becoming progressively rarer (Wilson et al., 2020).

We parametrize the effect size distribution as a mixture of two normal distributions. The first one, distribution $\mathrm{A}$, is centered in 0 , with standard deviation $\mathbf{S D}_{\mathrm{A}}$. The second one, distribution $\mathrm{B}$, is centered at $\boldsymbol{\mu}_{\mathbf{B}}$, with standard deviation $\mathbf{S D}_{\mathbf{B}}$. The relative weight of distribution $\mathrm{B}$ in the mixture is given by $\mathbf{W}_{\mathbf{B}}$, between 0 and 1 . From this parametrization, we define 5 types of distributions to model possible scenarios in different scientific fields (see Figure 2 for illustration and Table 2 for parameters).

The Dichotomous model is equivalent to that in Ioannidis (2005), where effects are dichotomized as true/false. Here, effects are either large or null - exactly 1 or exactly 0 -, approximating the Ioannidis (2005) model, in which the fact that power is fixed implicitly assumes that true effect sizes have similar magnitudes. The Two Peaks model is a more realistic dichotomous model, with Gaussian variation around means of 1 and 0 for true and null effects, respectively nevertheless, it still yields a clear distinction between both categories of effects. The Overlapping Peaks has peaks with a broader spread, with the two normal distributions showing a much greater degree of overlap - which effectively precludes defining them as containing "null" and "true" effects. In the Two Normals model, both normals are centered at 0 and differ only in their standard deviation. Our last scenario uses a Single Normal as the underlying distribution of effect sizes for simplicity and plausibility.

As effect sizes are modeled as continuous, calculating measures such as the false positive rate or the positive predictive value requires defining a minimum effect of interest to determine what constitutes a true effect - i.e. one whose absolute size is larger than the minimum. This parameter is also used for statistical power/sample size calculations (see below) and is set to 0.5 for all simulations. As the standard deviation for the samples is fixed at 1 (see below), effect sizes can be interpreted as Cohen's $d$, with our minimum corresponding to a medium effect size in Cohen's original classification (Cohen, 1977).

To allow the prevalence of true hypotheses - a key parameter in the Ioannidis (2005) model - to vary in models with two distributions, we change the relative weight of both distributions $\left(\mathbf{W}_{\mathbf{B}}\right)$ while maintaining the other parameters fixed. For the Single Normal model, in which $\mathbf{W}_{\mathbf{B}}$ is set to 0 , variation in the prevalence of true effects is achieved by changing the standard deviation, $\mathbf{S D}_{\mathrm{A}}$ (see Table 2). Importantly, this causes the range of achievable prevalences to vary from model to model, precluding simulation of some of the scenarios in Table 1 and Tables S1-S3. 


$\begin{array}{ccccc}\text { Scenario } & \mathbf{S D}_{\mathbf{A}} & \boldsymbol{\mu}_{\mathrm{B}} & \mathbf{S D}_{\mathbf{B}} & \mathbf{W}_{\mathbf{B}} \\ \text { Dichotomous } & 0 & 1 & 0 & \text { varies } \\ \text { Two Peaks } & 0.1 & 1 & 0.1 & \text { varies } \\ \text { Overlapping Peaks } & 0.3 & 1 & 0.3 & \text { varies } \\ \text { Two Normals } & 0.1 & 0 & 1 & \text { varies } \\ \text { Single Normal } & \text { varies } & 0 & 0 & 0\end{array}$

Table 2. Model parameters for different effect size distributions. Columns show parameters used for each version of the model. The weight of the second normal $\mathbf{W}_{\mathbf{B}}$ is varied to determine prevalence for the different simulations. When a single normal is used, this is accomplished by varying $\mathbf{S D}_{\mathbf{A}}$. For Figures $2-4, \mathbf{W}_{\mathbf{B}}$ was set to $0.1,0.3,0.5,0.7$ and 0.9 to achieve prevalences between 0.1 and 0.9 in the first 4 models. In the Single Normal model, $\mathbf{S D}_{\mathrm{A}}$ was set to $0.3,0.5,0.8$, 1.5 and 4 to approximate the same prevalences

\section{Sampling}

Once an effect size ES is investigated by a scientist, experimental samples are generated. For the control group, a sample of a fixed size is drawn from a normal distribution $\mathrm{N}(0,1)$. For the treatment group, a sample of the same size is drawn from a normal $\mathrm{N}(\mathbf{E S}, 1)$. Sample size is determined by setting statistical power to the desired value. Power is specified to detect a typical difference for the field, defined as the average of the effect sizes above the minimum of interest within the model's distribution of effects (by sampling 100,000 effects from the distribution under study). This means that in the Dichotomous and Two Peaks scenarios, statistical power refers to a difference very close to 1 . In the Single Normal model, as prevalence increases, the larger SD (up to 4) means that typical effect sizes become larger; therefore, power for a given effect size decreases as prevalence increases. To control for this fact, we also run simulations using the minimum of interest or twice its value for power calculations for this particular model in Figures S3 to S5.

Effects above 0.5 (i.e. true effects) lead to true-positive results when the observed effect is both significant and in the same direction as the real one, or to false negatives when a nonsignificant result is obtained. Effects below 0.5 (i.e. null effects) lead to true-negative results when the observed effect is non-significant or to false positives when a significant result is obtained in either direction, either through chance or bias. In cases where a significant estimate with the opposite signal of the actual effect is obtained, it is counted as a false positive. 


\section{Analysis and publication of results}

After sampling, a two-tailed Student's t test is performed to compare the two samples, assuming equal variances. If the result indicates a statistically significant difference at $\alpha=0.05$, the finding is published as the estimated difference between the means of the two groups. If the test does not return a significant result, one of two outcomes happens depending on bias probability $\mathbf{b}$ : either the result is not published with probability $1-\mathbf{b}$, or the experiment is repeated until the critical value for significance is reached in either direction and then published with probability $\mathbf{b}$. Both the assumption of publication bias and the implementation of bias represent simplified and pessimistic versions of the actual scientific process. Nevertheless, the bias term provides a simple way to model the outcome of both misconduct and questionable research practices, such as HARKing and flexibility of analysis, under the assumption that, with enough degrees of freedom, a significant p-value should be easy to find (Simmons et al., 2011).

Notice that the decision to publish in the main simulations does not take into account the minimum of interest - i.e. a significant result with a magnitude lower than 0.5 is still published. We believe this is a realistic assumption in basic biomedical science: as most articles do not present sample size calculations or discussions about effect sizes (Macleod et al., 2015), it seems reasonable to assume that they do not influence the decision to publish. Nevertheless, we include simulations where results are only published if they are significant and larger than the minimum of interest, which are presented in Figure S2.

\section{Evaluation of the generated literature}

Reported simulations consist of 5,000 published findings for each combination of parameters. For these findings, we compute different measures of accuracy: the positive predictive value (PPV) the type S error rate and the median type M error magnitude (Gelman \& Carlin, 2014).

The PPV is calculated as the percentage of positive findings in the literature that represent true positives, as defined by the minimum effect of interest. Since we assume complete publication bias, every published result is positive. Thus, true effects are defined as those whose true absolute value is larger than the minimum effect size of interest. Once again, we note that true positives are only counted as such if they have been estimated to have the same signal as the real effect.

The type S error rate is the proportion of significant estimates with the opposite signal of the real effect in the published literature, irrespective of them being above or below the minimum effect size of interest. Note that, as full publication bias is assumed, this will only happen when a significant estimate with the opposite signal is obtained. For published estimates that have the correct signal, we also compute the type $\mathrm{M}$ error, or exaggeration factor, by dividing the obtained 
estimate by the true effect size for each simulation. For each parameter combination, we report the median and interquartile range of the exaggeration factor for the simulated published literature.

\section{Source Code and Data}

The model was developed in R 3.6.3 (R Core Team, 2020). Code for the model, along with auxiliary scripts used to make graphs and analyses, are available on GitHub (https://github.com/KleberNeves/reproducibility-model). The generated data for the simulations shown here are available at https://osf.io/z2hn9/files/. The model is available as a Shiny App at https://kneves.shinyapps.io/repro-model/, where one can change the parameters described above (as well as others), run simulations and explore the results.

\section{Funding \& Acknowledgements}

This work was supported through grants from FAPERJ (E-26/203.222/2017) and the Serrapilheira Institute.

\section{Author Contribution Statement}

KN, PBT and OBA designed the research, analyzed the results and wrote the manuscript. KN implemented the code for the model and data analysis/visualization and wrote the original draft. OBA supervised the work.

\section{References}

Amaral, O. B., Neves, K., Wasilewska-Sampaio, A. P., \& Carneiro, C. F. (2019). The Brazilian Reproducibility Initiative. eLife, 8, e41602. https://doi.org/10.7554/eLife.41602

Baker, M. (2016). 1,500 scientists lift the lid on reproducibility. Nature News, 533(7604), 452. https://doi.org/10.1038/533452a

Bird, A. (2018). Understanding the replication crisis as a base rate fallacy. The British Journal for the Philosophy of Science. https://doi.org/10.1093/bjps/axy051

Carneiro, C. F., Moulin, T. C., Macleod, M. R., \& Amaral, O. B. (2018). Effect size and statistical power in the rodent fear conditioning literature - A systematic review. PloS One, 13(4), e0196258. https://doi.org/10.1371/journal.pone.0196258

Fidler, F., \& Cumming, G. (2014). Yes, but don’t underestimate estimation: Reply to Morey, Rouder, Verhagen, and Wagenmakers (2014). Psychological Science, 25(6), 1291-1292. https://doi.org/10.1177/0956797614532658

Cohen J. (1977). Statistical power analysis for the behavioral sciences ( $2^{\text {nd }}$ ed.). New York: Academic Press. 
Gelman, A. (2018). The failure of null hypothesis significance testing when studying incremental changes, and what to do about it. Personality and Social Psychology Bulletin, 44(1), 16-23. https:// doi.org/10.1177/0146167217729162

Gelman, A., \& Carlin, J. (2014). Beyond power calculations: Assessing Type S (Sign) and Type M (Magnitude) errors. Perspectives on Psychological Science, 9(6), 641-651.

https://doi.org/10.1177/1745691614551642

Goodman, S., \& Greenland, S. (2007). Assessing the unreliability of the medical literature: a response to "Why most published research findings are false". Johns Hopkins University, Dept. of Biostatistics Working Papers. Working Paper 135.

Ioannidis, J. P. A. (2005). Why most published research findings are false. PLoS Medicine, 2(8), e124. https://doi.org/10.1371/journal.pmed.0020124

Ioannidis, J. P. A. (2008). Why most discovered true associations are inflated. Epidemiology, 19(5), 640-648. https://doi.org/10.1097/EDE.0b013e31818131e7

Jamieson, K. H. (2018). Crisis or self-correction: Rethinking media narratives about the well-being of science. Proceedings of the National Academy of Sciences of the United States of America, 115(11), 2620-2627. https://doi.org/10.1073/pnas.1708276114

Kennedy-Shaffer, L. (2019). Before $\mathrm{p}<0.05$ to beyond $\mathrm{p}<0.05$ : Using history to contextualize pvalues and significance testing. The American Statistician, 73(sup1), 82-90.

https://doi.org/10.1080/00031305.2018.1537891

Mayo, D., \& Morey, R. D. (2017). A poor prognosis for the diagnostic screening critique of statistical tests. Open Science Framework Preprints. https://doi.org/10.31219/osf.io/ps38b

Macleod, M. R., McLean, A. L., Kyriakopoulou, A., Serghiou, S., de Wilde, A., Sherratt, N., ... \& Sena, E. S. (2015). Risk of bias in reports of in vivo research: a focus for improvement. PLoS Biology, 13(10), e1002273. https://doi.org/10.1371/journal.pbio.1002273

McShane, B. B., \& Gal, D. (2017). Statistical significance and the dichotomization of evidence. Journal of the American Statistical Association, 112(519), 885-895.

https://doi.org/10.1080/01621459.2017.1289846

McShane, B. B., Gal, D., Gelman, A., Robert, C., \& Tackett, J. L. (2019). Abandon statistical significance. The American Statistician, 73(sup1), 235-245.

https://doi.org/10.1080/00031305.2018.1527253

Meehl, P. E. (1978). Theoretical risks and tabular asterisks: Sir Karl, Sir Ronald, and the slow progress of soft psychology. Journal of Consulting and Clinical Psychology, 46(4), 806.

https://doi.org/10.1016/j.appsy.2004.02.001

Moonesinghe, R., Khoury, M. J., \& Janssens, A. C. J. (2007). Most published research findings are false-But a little replication goes a long way. PLoS Medicine, 4(2), e28.

https://doi.org/10.1371/journal.pmed.0040028

Open Science Collaboration. (2015). Estimating the reproducibility of psychological science.

Science, 349(6251), aac4716-aac4716. https://doi.org/10.1126/science.aac4716

Orben, A, \& Lakens, D. (2020). Crud (re)defined. Advances in Methods and Practices inPpsychological Science, 3(2), 238-247. https://doi.org/10.1177/2515245920917961 
Protzko, J., Krosnick, J., Nelson, L. D., Nosek, B. A., Axt, J., Berent, M., ... \& Schooler, J. (2020). High replicability of newly-discovered social-behavioral findings is achievable. PsyArXiv. https://doi.org/10.31234/osf.io/n2a9x

R Core Team. (2020). R: A language and environment for statistical computing. R Foundation for Statistical Computing. Retrieved from https://www.R-project.org/

Simmons, J. P., Nelson, L. D., \& Simonsohn, U. (2011). False-positive psychology: Undisclosed flexibility in data collection and analysis allows presenting anything as significant. Psychological Science, 22(11), 1359-1366. https://doi.org/10.1177/0956797611417632

Stanley, D. J., \& Spence, J. R. (2014). Expectations for replications: Are yours realistic? Perspectives on Psychological Science, 9(3), 305-318. https://doi.org/10.1177/1745691614528518 Steinfath, M., Vogl, S., Violet, N., Schwarz, F., Mielke, H., Selhorst, T., Greiner, M., \& Schönfelder, G. (2018). Simple changes of individual studies can improve the reproducibility of the biomedical scientific process as a whole. PLoS One, 13(9), e0202762.

https://doi.org/10.1371/journal.pone.0202762

Stewart, A. J., \& Plotkin, J. B. (2020). The natural selection of good science. arXiv:2003.00928. https://arxiv.org/abs/2003.00928

Szucs, D., \& Ioannidis, J. P. (2017). Empirical assessment of published effect sizes and power in the recent cognitive neuroscience and psychology literature. PLoS Biology, 15(3), e2000797. https://doi.org/10.1371/journal.pbio.2000797

Ulrich, R., \& Miller, J. (2020). Meta-Research: Questionable research practices may have little effect on replicability. eLife, 9, e58237. https://doi.org/10.7554/eLife.58237

van Zwet, E., \& Cator, E. (2020). The significance filter, the winner's curse and the need to shrink. arXiv:2009.09440. https://arxiv.org/abs/2009.09440

Wilson, B. M., Harris, C. R., \& Wixted, J. T. (2020). Science is not a signal detection problem. Proceedings of the National Academy of Sciences of the United States of America, 117(11):55595567. https://doi.org/10.1073/pnas.1914237117 